\title{
Anaesthetic Factors Affecting Outcome After Bariatric Surgery, a Retrospective Levelled Regression Analysis
}

\author{
Jan P. Mulier ${ }^{1,2,3}$ (D) Bruno Dillemans ${ }^{4}$
}

Published online: 16 March 2019

(C) The Author(s) 2019

\begin{abstract}
Background Deep neuromuscular block (NMB) and opioid-free anaesthesia (OFA) improve surgical workspace and reduce postoperative opioid consumption, but its impact on perioperative outcomes is unknown. This observational study compared complications and healthcare resource utilization after bariatric surgery, with or without continuous deep NMB or OFA.

Methods We included all 9246 patients who underwent laparoscopic bariatric surgery at our institution from January 2009 to February 2017. Continuous clinical deep NMB was defined as receiving a continuous infusion of rocuronium with a dose of $>$ $1 \mathrm{mg} / \mathrm{kg} \mathrm{IBW}$ for each hour or sugammadex $>2 \mathrm{mg} / \mathrm{kg}$ total body weight at the time of reversal. We analysed the effect of continuous clinical deep NMB and OFA and covariates on 1 month post-operative complications using the Clavien-Dindo (CD) classification (grades II-V) and healthcare utilization (hospital length of stay [LOS], rates of reoperations within 1 week, highdependency care unit admissions, and readmissions within 1 month). Covariates included experience of the attending anaesthesiologist, patient age, sex, body mass index, American Society of Anesthesiologists physical status score, obstructive sleep apnoea syndrome, diabetes, hypertension, surgery type, surgical team experience, and neostigmine use.

Results OFA, continuous deep NMB, surgical and anaesthesia team experience, younger age, and surgery type were associated with fewer complications. OFA was associated with lower healthcare resource utilization. Reduced LOS was also associated with younger age, surgical team experience, and surgery type, but not continuous deep NMB.

Conclusion Continuous deep NMB and OFA were associated with fewer complications after bariatric surgery.
\end{abstract}

Keywords Deep neuromuscular block $\cdot$ Laparoscopy $\cdot$ Bariatric surgery $\cdot$ Outcome $\cdot$ Opioid-free anaesthesia

\section{Background}

Bariatric surgery outcomes have improved. The combination of quality and high volume allowed for surgical standardization

Jan P. Mulier

jan.mulier@azsintjan.be; janpaul.mulier@gmail.com

Bruno Dillemans

bruno.dillemans@azsintjan.be

1 Department of Anesthesiology, Intensive Care and Reanimation, AZ Sint Jan Brugge-Oostende, Ruddershove 10,

B-8000 Brugge, Belgium

2 Department of Anesthesiology, KU Leuven - University of Leuven, B-3000 Leuven, Belgium

3 Department of Anesthesiology, UGent - University of Gent, B-9000 Gent, Belgium

4 Department of General Surgery, AZ Sint Jan Brugge-Oostende, B-8000 Brugge, Belgium with impressive results [1]. Since 2009 at the bariatric centre of the AZ Sint Jan Brugge-Oostende, there have been no leaks after primary gastric bypass or sleeve procedures, and surgical revisions for post-operative bleeding dropped to $<0.1 \%$. Anaesthesia advancements allowed for strong leak tests and intra-operative blood pressure control to improve surgical outcomes. For example, instead of slowly injecting $50 \mathrm{ml}$ of methylene blue water, Mulier et al. injected $150 \mathrm{ml}$ of methylene blue water with chlorhexidine as quickly as possible through a 34 or 40 French gauze gastric tube followed by $150 \mathrm{ml}$ of air to stress the surgical staple lines intra-operatively to find the weakest spots that would require extra stitches to prevent leakage. Increasing the systolic pressure above the normal values of $140 \mathrm{mmHg}$ revealed arterial sprayers that would have otherwise been missed and might have continued to bleed postoperatively each time the blood pressure rose.

Anaesthesia for bariatric surgery changed after 2009 in our bariatric centre with the foundation of the European Society for Perioperative Care (ESPCOP) and the leading teaching 
role that followed, and more attention was paid to safer anaesthesia induction, silent aspiration prevention, and other small details, which we assume has gradually improved outcomes. Continuous deep neuromuscular block (NMB) and opioidfree anaesthesia (OFA) were introduced in 2009, first in a limited number of cases, and then for around $50 \%$ of all cases. NMB improves surgical workspace whereas OFA enhances recovery, and both may therefore improve surgical outcomes.

Deep NMB is frequently requested by bariatric surgeons when performing laparoscopic surgery in morbidly obese patients [2]. In 2015, Madsen et al. [3] recommended deep NMB during certain laparoscopic procedures based on their systematic review, but these authors did not address bariatric procedures or post-operative outcomes. Mulier et al. [4] reported that only deep NMB using rocuronium lowered intraabdominal pressure (IAP) at zero volume insufflation and increased the objective measured laparoscopic workspace, compared with sevoflurane or remifentanil. These changes improved surgical access or allowed lower insufflation pressures. In 2017, Bruintjes et al. [5] reported in their meta-analysis that deep NMB improves laparoscopic surgical space and reduces post-operative pain. Continuous clinical deep NMB until the end of surgery also prevents sudden muscle movements, which are dangerous and can cause intra-operative surgical mishaps [6]. A lower IAP with deep NMB may also reduce peritoneal ischemia and inflammatory reactions [7].

Obese patients with obstructive sleep apnoea syndrome (OSAS) are at higher risk for obstructed breathing, and can post-operatively require continuous positive airway pressure (CPAP) masks and admission to highdependency care. However, admitting every patient with OSAS would create logistic problems. Enhanced recovery after surgery (ERAS) suggested to maximally reducing post-operative opioid use to prevent all opioid-related side effects [8]. Multimodal analgesia does not significantly reduce opioid use. General anaesthesia combined with epidural block is a possible solution, but may be too aggressive and potentially dangerous in morbidly obese patients undergoing a laparoscopic procedure. Therefore, a new, less invasive method was required that would consistently reduce post-operative opioid use.

Tolerance and hyperalgesia result in a high post-operative opioid requirement even after a less invasive laparoscopic procedure [9]. The ERAS society suggests the use of shortacting opioids intra-operatively to rapidly decrease circulating opioids, but more opioids are required post-operatively, which negates the desired effect. After total intravenous anaesthesia (TIVA), patients typically exhibit more tolerance and hyperalgesia, therefore requiring a higher dose of opioids post-operatively.

Unfortunately, tolerance for respiratory and gastrointestinal depression takes much longer to develop than a tolerance for analgesia [10], and the risk of side effects like hypoventilation and post-operative nausea and vomiting (PONV) increases linearly with the logarithmic dose of opioids given [11].

Several additives when given intravenously (iv), such as alpha2agonists, ketamine, or local anaesthetics, can intraoperatively reduce the necessary opioid dose. When we used a combined multitarget approach, we reduced intra-operative opioid use to zero while keeping the patient stress-free and still allowing for rapid awakening after anaesthesia. After an OFA, we found that the post-operative opioid dose could easily be reduced by $75 \%$.

Deep NMB clearly facilitates laparoscopic work and OFA improves recovery [12], but it is not clear if these techniques also lead to fewer perioperative complications. Given the low number of major post-operative complications, a randomized trial would be too small to answer this question.

The primary objective of this study was to describe the relationship of anaesthetic management to the rate of complications within 1 month of surgery, as categorized by the Clavien-Dindo (CD) scale (excepting grade 1 minor complications) using a single-centre cohort study of patients undergoing bariatric surgery. The secondary objective was to describe how healthcare resources were utilized by these patients, by examining the rate of surgical reoperation within 1 week, rate of high-dependency unit admissions, rate of readmissions to the hospital within 1 month, and hospital length of stay (LOS).

\section{Methods}

Ethics approval for this retrospective study was provided by the hospital Ethical Committee of AZ Sint Jan BruggeOostende. We included all patients in our hospital database who underwent laparoscopic bariatric surgery between January 2009 and February 2017. The study was registered on Clinicaltrials.gov with the identifier: NCT03200964. All patients provided consent to allow retrospective data analysis without patient identification.

Patients were classified as receiving OFA when no opioids were given pre- or intra-operatively until wound closure. Opioids given after wound closure were counted as postoperative opioids. Patients who received a lower dose of intra-operative opioids by using additives were still classified as opioid anaesthesia (OA). Post-operative OFA was classified as receiving no opioids after wound closure until discharge from hospital in patients not receiving medium- or longacting opioids, pre- or intra-operatively.

The method of reaching OFA changed over the years. In 2011, OFA was first achieved by using clonidine, lidocaine, and esmolol. In 2012, dexmedetomidine was used in a loading dose of $1 \mathrm{mcg} / \mathrm{kg}$ ideal body weight (IBW) and an infusion of $1 \mathrm{mcg} / \mathrm{kg}$ IBW/h, which replaced clonidine and esmolol. In 2013, low-dose ketamine (initially $10 \mathrm{mg}$, but later increased 
to $50 \mathrm{mg}$ ) and magnesium sulphate $(40 \mathrm{mg} / \mathrm{kg}$ IBW) were added. This multitarget approach reduced the dexmedetomidine dose to an average of $0.6 \mathrm{mcg} / \mathrm{kg}$ IBW in 2015, resulting in a bariatric procedure with improved postoperative waking with less sedation. Post-operative analgesia was further improved by continuing very low doses of dexmedetomidine $(0.05 \mathrm{mcg} / \mathrm{kg} \mathrm{IBW} / \mathrm{h})$, ketamine $(0.05 \mathrm{mg} / \mathrm{kg} \mathrm{IBW} / \mathrm{h})$, and lidocaine $(0.5 \mathrm{mg} / \mathrm{kg} \mathrm{IBW} / \mathrm{h})$ for the first hours (maximum $5 \mathrm{~h}$ ) with the possibility of giving a bolus of $10 \mathrm{mg}$ lidocaine, $1 \mathrm{mg}$ ketamine, and $1 \mathrm{mcg}$ dexmedetomidine. Beginning in 2016, procaine was administered after this infusion for the next $10 \mathrm{~h}$, further reducing the post-operative use of rescue opioids. Some anaesthesiologists also intra-operatively added one or more additives such as clonidine, ketamine, or lidocaine, which also reduced the intra-operative dose of sufentanil over time.

We classified patients as receiving continuous clinical deep NMB if they received a continuous infusion of rocuronium with a dose of $>1 \mathrm{mg} / \mathrm{kg}$ IBW for each hour of surgery or a sugammadex dose of $>2 \mathrm{mg} / \mathrm{kg}$ total body weight (TBW) (based on a train of four count of 0/4) at the time of reversal.

Perioperative complications during the first post-operative month were graded according to the modified CD scale, with the exclusion of minor grade 1 complications. The $\mathrm{CD}$ grading allowed us to rank severity from 2 to 7 , which was used in the statistical analysis by giving grade 2 a ranking of 2 , grade $3 \mathrm{a}$ a ranking of 3 , grade $3 \mathrm{~b}$ a ranking of 4 , grade $4 \mathrm{a}$ a ranking of 5 , and grade $4 \mathrm{~b}$ a ranking of 6 . We also added a rank 7 to represent the deaths. The disability grading $\mathrm{d}$ was not added to every level because it did not allow for ranking for statistical analysis.

Anaesthetic experience was determined by the number of anaesthetics administered for bariatric procedures in the last 12 months by each anaesthesiologist or resident (under supervision). This allowed us to perform a levelled regression analysis using anaesthesiologist experience as a level instead of a covariate.

We analysed the differences in healthcare resource utilization between groups by measuring the number of patients who required admission to a high-dependency care unit, number of patients who required surgical reoperation during the first week after surgery, hospital LOS calculated as the total number of nights in hospital, and frequency of readmission to the hospital within 1 month after surgery.

We retrieved all measured factors potentially related to complications of grade II to grade V or healthcare utilization outcomes from the database and medical records. Surgical team experience was measured according to the year in which the procedure was performed, assuming that experience for the total team changed over the years. OSAS was defined as a diagnosis of sleep apnoea confirmed by polysomnography and requiring CPAP therapy or a STOP-Bang score $>5$, indicating high risk for OSAS
[13]. Post-operative opioids were defined as the total dose of opioids used during the first $24 \mathrm{~h}$ post-operatively, calculated as iv morphine equivalents. The following were considered equivalent to $1 \mathrm{mg}$ iv morphine: $1 \mathrm{mg}$ iv or subcutaneous piritramide, $10 \mathrm{mg}$ iv tramadol, or $2 \mathrm{mg}$ sublingual oxycodone [14].

Deep NMB can only be reversed by sugammadex, whereas a moderate block can be reversed by sugammadex or neostigmine. Because of the possible impact of neostigmine on outcome, we also examined the use of neostigmine over the study period as a cofactor.

\section{Statistical Analysis}

We performed a levelled linear regression analysis using anaesthesia experience as level and the CD ranking as outcome. We used the following cofactors to evaluate their effect on the outcome measures: year of surgery as a measure of surgical experience, age, gender, preoperative existence of hypertension, severe OSAS, ASA physical status, conversion procedure after previous bariatric procedure, use of neostigmine, OFA, and continuous clinical deep NMB until the end of surgery. A $p$ value $<0.05$ was considered statistically significant.

The levelled linear regression analysis used the same cofactors each time to also describe the effect on reoperation, high-dependency admission, readmission, and hospital LOS.

\section{Results}

There were a total of 9246 surgical procedures recorded between January 2009 and February 2017. Fifty patients were excluded due to insufficient data.

The number of primary gastric bypass operations, conversion procedures, and sleeve gastrectomies is shown in Table 1. The numbers of patients receiving OFA or continuous clinical deep NMB are shown in Table 2.

The demographic and clinical characteristics of continuous deep NMB vs. non-continuous deep NMB groups are shown in Table 3.

The continuous deep NMB group had a higher proportion of women, older patients, patients with hypertension, and patients who underwent sleeve surgery or OFA. They had a lower rate of conversion surgery and lower postoperative opioid use.
Table 1 Number of different surgical procedures 
Table 2 Number of different anaesthesia procedures

Opioid-free anaesthesia/opioid anaesthesia

$2966 / 6230$

Continuous clinical deep NMB/no deep NMB

$1833 / 7363$

Missing information

50

Total

9246

The demographic and clinical characteristics of OFA vs. OA groups are shown in Table 4.

The OFA group had a higher proportion of older patients, more OSAS, more conversion procedures, and more continuous deep NMB. OFA-treated patients had a lower BMI and received fewer sleeve procedures.

The number of recorded complications in each CD group, excluding grade I complications, is shown in Table 5 for the continuous clinical deep NMB group, for the OFA group, and for the remaining non-continuous clinical deep NMB and OA group. We did not record grade I complications. Grade II represents the minor complications, whereas grades III, IV, and V represent major complications. Some patients received OFA combined with continuous clinical deep NMB.

The percentage of patients receiving OFA or continuous clinical deep NMB from 2009 to 2017 and the total percentage of major complications according to $\mathrm{CD}$ grading $(\mathrm{CD}>\mathrm{II})$ are shown in Fig. 1.

The percentage of patients with major or minor pulmonary complications in the OFA or OA groups is shown in Fig. 2.
The number of patients each year who were reversed from moderate NMB with neostigmine or sugammadex and reversed from deep NMB is shown in Fig. 3.

Table 6 shows the levelled logistic regression analysis using anaesthesia experience as level 1 for CD graded complications, reoperation, admission to high-dependency care, readmission, and hospital length of stay.

We found that OFA, continuous clinical deep NMB, greater surgical team experience, younger patient age, and the absence of a conversion procedure were independently associated with fewer CD complications. OFA was associated with lower utilization of healthcare resources. The total numbers in the OFA and OA groups are shown in Table 7.

Reduced LOS was also associated with younger patient age, greater surgical team experience, and the absence of a conversion procedure, but not with use of a continuous clinical deep NMB. Neostigmine use was not associated with complications.

Patients with a complication $(\mathrm{CD}>1)$ perioperatively received significantly more morphine equivalents in the OFA $(8.68 \pm 1.91$ vs $3.34 \pm 0.25 p=0.001)$ and $\mathrm{OA}(45.27 \pm 3.27$ vs $34.61 \pm 1.09 p=0.001)$ groups. The dose of opioids was significantly lower $(p=0.001)$ for patients with and without complications in the OFA group.

Intra-operative use of opioids (99\% sufentanil) declined from around 40 to $30 \mathrm{mcg}$ in the OA group before complete OFA introduction, but remained stable after 2012. Post-operative opioid use also declined until 2012,
Table 3 Demographic and clinical characteristics of the patients who received deep vs. non-deep NMB

\begin{tabular}{lllll}
\hline & $\begin{array}{l}\text { ContDeep } \\
\text { NMB } \\
(n=1833)\end{array}$ & $\begin{array}{l}\text { Non-ContDeep } \\
\text { NMB } \\
(n=7363)\end{array}$ & $p$ value & Test \\
& 22.6 & 27.9 & & \\
\hline Male (\%) & $42.47+0.58$ & $41.33+0.28$ & $<0.001$ & $t$ test \\
Age (years) (mean + SD) & $113.94+1.10$ & $117.69+0.58$ & $<0.001$ & $t$ test \\
Weight (mean + SD) & $166.53+0.47$ & $168.78+0.28$ & $<0.001$ & $t$ test \\
Length (cm) (mean + SD) & $40.92+0.31$ & $41.17+0.16$ & 0.178 & $t$ test \\
BMI (mean + SD) & 14.1 & 12.9 & 0.171 & $z$-score \\
Diabetes (\%) & 33.6 & 30.5 & 0.007 & $z$-score \\
Hypertension (\%) & $2.0+0.0$ & $1.9+0.0$ & $<0.001$ & $t$ test \\
ASA score (mean + SD) & 47.9 & 41.6 & 0.467 & $z$-score \\
OSAS (mean + SD) & 10.2 & 13.8 & 0.002 & $z$-score \\
Conversion procedure (\%) & 9.3 & 3.2 & $<0.001$ & $z$-score \\
Type of surgery (\% sleeve) & 65.2 & 15.7 & $<0.001$ & $z$-score \\
Type of anaesthesia (\% OFA) & $3.72+0.31$ & $5.61+0.33$ & $<0.001$ & $t$ test \\
Total dose of morphine equivalents post-operative & & & & \\
$\quad$ (mg) (mean + SD) & & & &
\end{tabular}

ASA, American Society of Anesthesiologists; BMI, body mass index; ContDeep NMB, continuous deep neuromuscular blockade; OFA, opioid-free anaesthesia; OSAS, obstructive sleep apnoea syndrome; SD, standard deviation 
Table 4 Demographic and clinical characteristics of the patients who received OFA vs. $\mathrm{OA}$

\begin{tabular}{lllrl}
\hline & $\begin{array}{l}\text { OFA } \\
(n=2966)\end{array}$ & $\begin{array}{l}\text { OA } \\
(n=6230)\end{array}$ & $p$ value & Test \\
\hline Male (\%) & 28.0 & 27.3 & 0.531 & $z$-score \\
Age (years) (mean + SD) & $42.33+0.51$ & $41.19+0.34$ & $<0.001$ & $t$ test \\
Weight (mean + SD) & $116.074+0.84$ & $117.21+0.64$ & 0.039 & $t$ test \\
Length (cm) (mean + SD) & $168.42+0.36$ & $168.37+0.31$ & 0.829 & $t$ test \\
BMI (mean + SD) & $40.92+0.31$ & $41.17+0.16$ & 0.009 & $t$ test \\
Diabetes (\%) & 13.7 & 12.8 & 0.283 & $z$-score \\
Hypertension (\%) & 31.6 & 30.0 & 0.163 & $z$-score \\
ASA score (mean + SD) & $2.32+0.035$ & $2.33+0.0024$ & 0.857 & $t$ test \\
OSAS (mean + SD) & 49.8 & 41.9 & 0.001 & $z$-score \\
Conversion procedure (\%) & 19.3 & 15.1 & 0.001 & $z$-score \\
Type of surgery (\% sleeve) & 1.0 & 4.2 & $<0.001$ & $z$-score \\
ContDeep NMB (\%) & 52.4 & 3.20 & $<0.001$ & $z$-score \\
Total dose of morphine equivalents post-operative & $3.407+0.236$ & $15.28+0.606$ & $<0.001$ & $t$ test \\
$\quad(m g)(m e a n+$ SD) & & & & \\
\hline
\end{tabular}

$p<0.05$ between groups

Data are number (\% of complication group)

Fifty patients were excluded because of inadequate data

ASA, American Society of Anesthesiologists; BMI, body mass index; ContDeep NMB, continuous deep neuromuscular blockade; OFA, opioid-free anaesthesia; OA, opioid anaesthesia; OSAS, obstructive sleep apnoea syndrome; SD, standard deviation and then remained around $15 \mathrm{mg}$ with no patients postoperatively receiving opioid-free analgesia as it is defined as no opioid use intra- or post-operative.

Post-operative opioid use in the OFA group declined rapidly in 2013 , with $30 \%$ of patients post-operatively receiving opioid-free analgesia. When intra-operative use of a high dose of dexmedetomidine was reduced in 2015, fewer patients received opioid-free analgesia post-operatively. In the last few years, the number of opioid-free analgesia patients has again increased, due to continuing use of an infusion of dexmedetomidine, lidocaine, and ketamine at very low dose post-operatively (Fig. 4).

\section{Discussion}

We used a retrospective cohort study instead of a randomized controlled study (RCT) to include every patient who received a bariatric procedure over a longer period. Our bariatric centre primarily performed Roux-en-Y gastric bypass procedures and small number of sleeve gastrectomies. However, a large number of conversion procedures were also included, which included conversions from a band to a bypass and reinterventions after a failed bypass.

In 2009, all anaesthesia procedures were balanced anaesthesia using inhalation and sufentanil with short periods of clinical
Table 5 Number of complications graded by ClavienDindo scale in patients who received continuous clinical deep $\mathrm{NMB}, \mathrm{OFA}$, and the remaining patients

\begin{tabular}{llll}
\hline $\begin{array}{l}\text { Number of complications graded by } \\
\text { Clavien-Dindo }\end{array}$ & $\begin{array}{l}\text { ContDeep NMB } \\
(n=1878)\end{array}$ & $\begin{array}{l}\text { OFA } \\
(n=2966)\end{array}$ & $\begin{array}{l}\text { Opioid and non-ContDeep } \\
\text { NMB }(n=6018)\end{array}$ \\
\hline CD II (minor complications) & $16(0.81 \%)$ & $29(0.98 \%)$ & $112(1.86 \%)$ \\
CD III & 7 & 10 & 13 \\
CD IIIb & 2 & 6 & 37 \\
CD IVa & 0 & 0 & 4 \\
CD IVb & 0 & 0 & 2 \\
CD V & 0 & 0 & 1 \\
Total number of major complications & $9(0.48 \%)$ & $16(0.54 \%)$ & $57(0.95 \%)$ \\
CD $>$ II & & & \\
\hline
\end{tabular}

There is overlap between patients getting ContDeep NMB and OFA, CD I is not recorded

ContDeep NMB, continuous deep neuromuscular blockade; OFA, opioid-free anaesthesia; OA, opioid anaesthesia 
Fig. 1 Number of patients receiving opioid-free anaesthesia, continuous clinical deep NMB from 2009 to 2017 , and the total percentage of major complications according to the ClavienDindo $(\mathrm{CD})$ grading $(\mathrm{CD}>\mathrm{II})$ $\%$ OFA $\%$ ctu deep NMB $\rightarrow \%$ Major complications each year $100 \%$ $2,0 \%$

$75 \%$

$50 \%$

$25 \%$
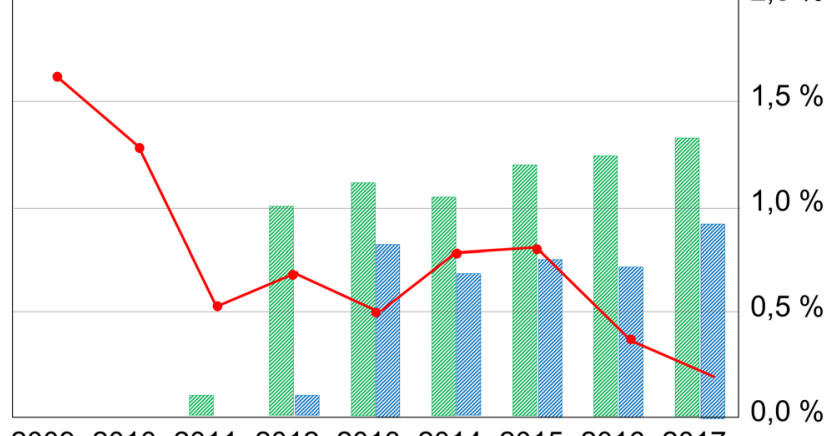

$0 \%$

200920102011201220132014201520162017 deep NMB when required. OFA began in 2011 and continuous clinical deep NMB in 2012. Each of the two procedures started with a small number of patients in the first year, depending on the attending anaesthesiologist. In the following years, OFA was used for $55 \%$ of patients and continuous clinical deep NMB for $35 \%$. Again, this usage was not based on the patient condition or the surgical procedure, but on the availability of an anaesthesiologist experienced with OFA or deep NMB. We considered the year of surgery and anaesthesiologist experience as co-variables to exclude this unequal distribution.

We know already from small randomized controlled trials that OFA reduces the required post-operative opioid dose [15] and that deep NMB improves the surgical workspace [2], but it was not known whether these techniques had an impact on outcome. In 2009, the occurrence of complications was already very low in our centre compared with other centres [1]. This is because of a zero leak rate since 2010 (from

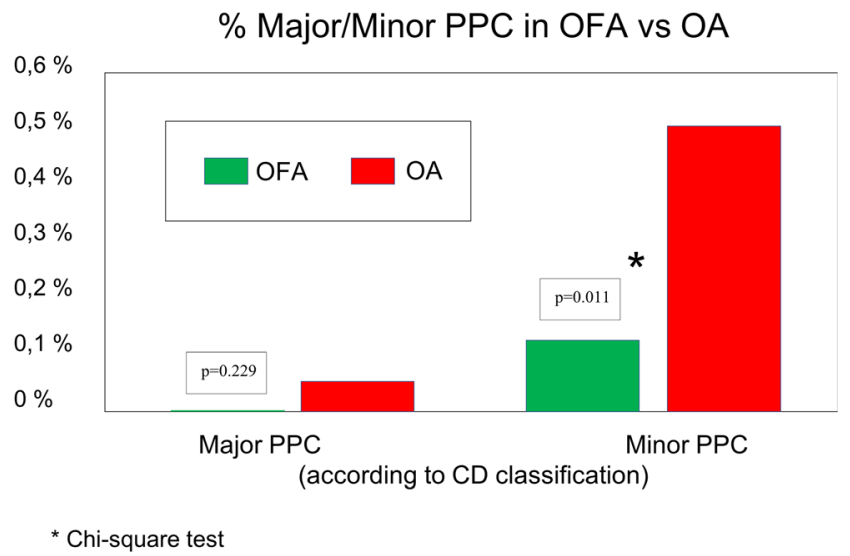

Fig. 2 The percentage of patients with a major or minor post-operative pulmonary complication (PPC) in the opioid-free (OFA) and in the opioid anaesthesia (OA) group
$0.12 \%$ in 2008 to $0 \%$ from 2010 till 2018 ) by many factors including performing strong leak tests intra-operative, and a dramatic reduction in revisions since 2009 (from $0.55 \%$ in 2008 to $0.10 \%$ in 2010 ) for post-operative bleeding by elevating the blood pressure at the end. Therefore, a randomized controlled study evaluating OFA or deep NMB would not show a difference between groups if less than 1000 patients were included. Further, few anaesthesiologists have the experience required to perform OFA or continuous deep NMB, making a very large multicentre randomized controlled trial of the same surgical procedures difficult. A retrospective cohort analysis cannot confirm a causal relationship, but a correlation can be made because all patients were not given an identical anaesthesia method. This retrospective cohort analysis improves the understanding of the combined impact of all efforts when a change in outcome is found.

We found no major pulmonary complications in the OFA group and only a limited number in the OA group. The total incidence of minor pulmonary complications was only $10 \%$ of all complications, and therefore insufficient to use as an outcome parameter. This finding is comparable to that of the Gallard study that found no increase in post-operative pulmonary complications (PPC) in obese patients [16].

We found that use of neostigmine for reversal gradually declined after 2009, when sugammadex became available (Fig. 3). In 2009, at our institution, we only used deep NMB for difficult cases with limited workspace, but did not continue using the technique to the end of surgery, so that we could reverse the $\mathrm{NMB}$ with a $2 \mathrm{mg} / \mathrm{kg}$ dose of sugammadex. In 2013, continuous clinical deep NMB until the end of laparoscopic surgery was used in approximately $1 / 3$ of cases, when the advantages became more obvious and we began to focus on the possibility of reducing peritoneal inflammation. The 
Fig. 3 Number of patients reversed from deep or moderate neuromuscular blockade with neostigmine or sugammadex from 2009 to 2016
Number of patients reversed with neostigmine or sugammadex

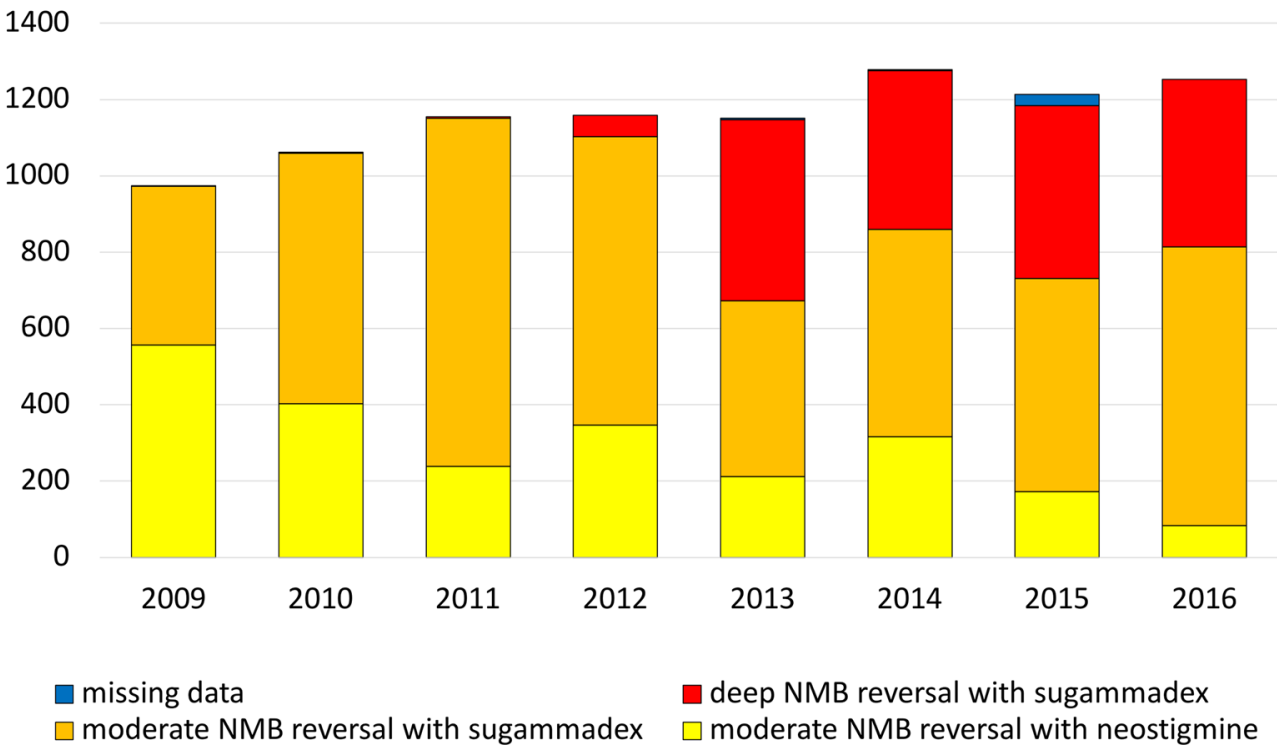

positive effects of deep NMB might further improve by using the lowest insufflation pressure possible.

The definition of deep NMB is a zero train of four (TOF) and a post-tetanic count (PTC) above zero [17]. This definition does not mention the muscle that should be monitored; however, a peripheral muscle like the thumb is more sensitive for NMB than a central muscle like the abdominal wall [18]. This definition of a deep NMB is not clinically useful because most patients will move or have contraction of their abdominal muscles during laparoscopy when PTC is more than 4. Therefore, a clinical deep NMB is defined as having a PTC between 0 and 4, assuming that a peripheral muscle is monitored [19]. In a continuous clinical deep NMB, the PTC should remain continuously at this value, never exceeding the count of 3 during surgery. However, this can be difficult to control, because PTC requires a manual command every 3 to $5 \mathrm{~min}$ in most neuromuscular transmission monitors [20]. In this retrospective cohort study, continuous measured PTC data were not available. Therefore, if the total dose of rocuronium was $>1 \mathrm{mg} / \mathrm{kg} / \mathrm{h}$ or if the patient had a clinical deep NMB at the moment of reversal, we assumed that the patient remained under continuous clinical deep NMB. Most anaesthesiologists

Table 6 Levelled logistic regression analysis using anaesthesia experience as level 1 for Clavien-Dindo graded complications, reoperation, highdependency care admission, readmission, and hospital length of stay

\begin{tabular}{|c|c|c|c|c|c|c|c|c|c|c|}
\hline \multirow[t]{2}{*}{ Factor } & \multicolumn{2}{|c|}{ Complications } & \multicolumn{2}{|c|}{ Reoperation } & \multicolumn{2}{|c|}{ High-dependency care unit admission } & \multicolumn{2}{|c|}{ Readmission } & \multicolumn{2}{|c|}{ Hospital length of stay } \\
\hline & $B$ coef & $P$ & $B$ coef & $P$ & $B$ coef & $P$ & $B$ coef & $P$ & $B$ coef & $P$ \\
\hline Anaesthesia experience & -0.25 & $<0.001$ & -0.56 & 0.318 & -0.748 & $<0.001$ & -0.16 & 0.275 & -0.162 & 0.311 \\
\hline Surgical team experience & -0.604 & $<0.001$ & -0.431 & 0.048 & -0.559 & $<0.001$ & 0.209 & 0.143 & -0.049 & 0.002 \\
\hline Age & 0.034 & $<0.001$ & 0.007 & 0.755 & 0.034 & 0.023 & 0.024 & 0.093 & 0.021 & $<0.001$ \\
\hline OFA & -0.075 & $<0.001$ & -0.034 & 0.001 & -0.043 & $<0.001$ & -0.024 & 0.005 & -0.005 & 0.002 \\
\hline Sex & -0.060 & 0.792 & -0.356 & 0.570 & 0.702 & 0.076 & 0.085 & 0.829 & -0.049 & 0.203 \\
\hline Hypertension & -0.017 & 0.929 & -0.537 & 0.371 & 0.176 & 0.615 & 0.008 & 0.982 & 0.054 & 0.191 \\
\hline Conversion & 0.624 & 0.003 & -0.208 & 0.753 & 0.557 & 0.164 & 0.373 & 0.297 & 0.448 & $<0.001$ \\
\hline ASA physical status & 0.061 & 0.639 & -0.347 & 0.279 & 0.129 & 0.636 & -0.391 & 0.059 & 0.268 & $<0.001$ \\
\hline OSAS & 0.295 & 0.215 & 0.926 & 0.161 & 0.832 & 0.063 & 0.225 & 0.587 & 0.242 & $<0.001$ \\
\hline ContDeep NMB & -0.608 & 0.020 & -1.027 & 0.192 & -0.922 & 0.096 & 0.122 & 0.713 & -0.061 & 0.125 \\
\hline Neostigmine & 0.1 & 0.591 & 1.054 & 0.026 & 0.422 & 0.206 & -0.031 & 0.0924 & -0.196 & $<0.001$ \\
\hline
\end{tabular}

$p<0.05$

Levelled logistic regression

ASA, American Society of Anesthesiologists; coef, coefficient; ContDeep NMB, continuous deep neuromuscular blockade; OFA, opioid-free anaesthesia; OSAS, obstructive sleep apnoea syndrome 
Table 7 Healthcare resource utilization after laparoscopic bariatric surgery in patients who did or did not receive opioid-free anaesthesia

\begin{tabular}{lllrl}
\hline & $\begin{array}{l}\text { OFA } \\
(n=2986)\end{array}$ & $\begin{array}{l}\text { OA } \\
(n=6202)\end{array}$ & $p$ value & Test \\
\hline LOS (days) (mean $\pm \mathrm{SD})$ & $2.56 \pm 0.04$ & $3.32 \pm 0.036$ & $<0.001$ & $t$ test \\
First week reoperation $(N, \%)$ & $6(0.2)$ & $33(0.9)$ & 0.002 & $z$-score \\
Readmission $(N, \%)$ & $29(1.1)$ & $65(1.1)$ & 0.993 & $z$-score \\
High-dependency admission $(N, \%)$ & $22(0.7)$ & $85(1.4)$ & 0.185 & $z$-score \\
\hline
\end{tabular}

$p<0.05$

ContDeep NMB, continuous deep neuromuscular blockade; LOS, length of stay; SD, standard deviation; OFA, opioid-free anaesthesia; OA, opioid anaesthesia. use only bolus doses for primary bariatric procedures and frequently wait until the train of four reaches 1 or until surgical conditions deteriorate before administering a second dose. The addition of magnesium as a standard drug in the OFA [21] prolongs deep NMB easier. Central muscles reverse 10 min earlier than peripheral muscles like the thumb [22]. The author suggests therefore a continuous infusion of rocuronium to facilitate a continuous clinical deep NMB. Because there is large variability in rocuronium's effects [23], it is possible that our definition of continuous clinical deep NMB according to rocuronium dose was not accurate for every patient, but was better than solely relying on the use of $>2 \mathrm{mg} / \mathrm{kg}$ TBW sugammadex for reversal [7]. Our definition indicated that NMB was deep at the moment of reversal, but did not exclude the possibility of short periods of moderate NMB during the procedure. Furthermore, continuous clinical deep NMB may be present until fascial closure, but could have become moderate by the time of reversal. Despite these limitations, our definitions of continuous clinical deep NMB, which combined the total administered doses of rocuronium and sugammadex, allowed us to clinically evaluate all patients undergoing bariatric surgery since 2009 .

Surgeons have more laparoscopic workspace when deep NMB is used, which is especially beneficial in obese patients [2]. Most anaesthesiologists at our institution do not take advantage of the ability to reduce IAP with deep $\mathrm{NMB}$, and the actual IAP during surgery is not routinely recorded. Usually, the surgeon begins with an insufflation pressure of $15 \mathrm{mmHg}$. If space is inadequate, the surgeon will first request verification of deep NMB before increasing the IAP to 18 or $20 \mathrm{mmHg}$ [24]. If space at first insufflation is sufficient, it is usually left to the anaesthesiologist to use a lower IAP after the trocars are positioned. Consequently, in most patients who receive deep NMB, the IAP might not have been decreased. Maintaining continuous clinical deep NMB by using an infusion of a neuromuscular blocking agent depended on the anaesthesiologist involved. This variability explains the higher incidence of OFA during deep NMB. Medium and longacting opioids given before the end of surgery remained active during recovery, but these were not considered post-operative opioids. Nevertheless, post-operative opioid consumption remained lower when OFA was used.

The finding that fewer male patients received continuous clinical deep NMB may reflect their greater height $(177.43 \mathrm{~cm}$ vs. $164.85 \mathrm{~cm} p<0.001$ ) and higher IBW calculation for rocuronium dosage. Indeed, patients who received continuous clinical deep NMB were significantly shorter than those in the
Fig. 4 Perioperative use of opioids over the years in opioid anaesthesia (OA) and opioid-free anaesthesia (OFA) in the bariatric centre of Bruges, Belgium

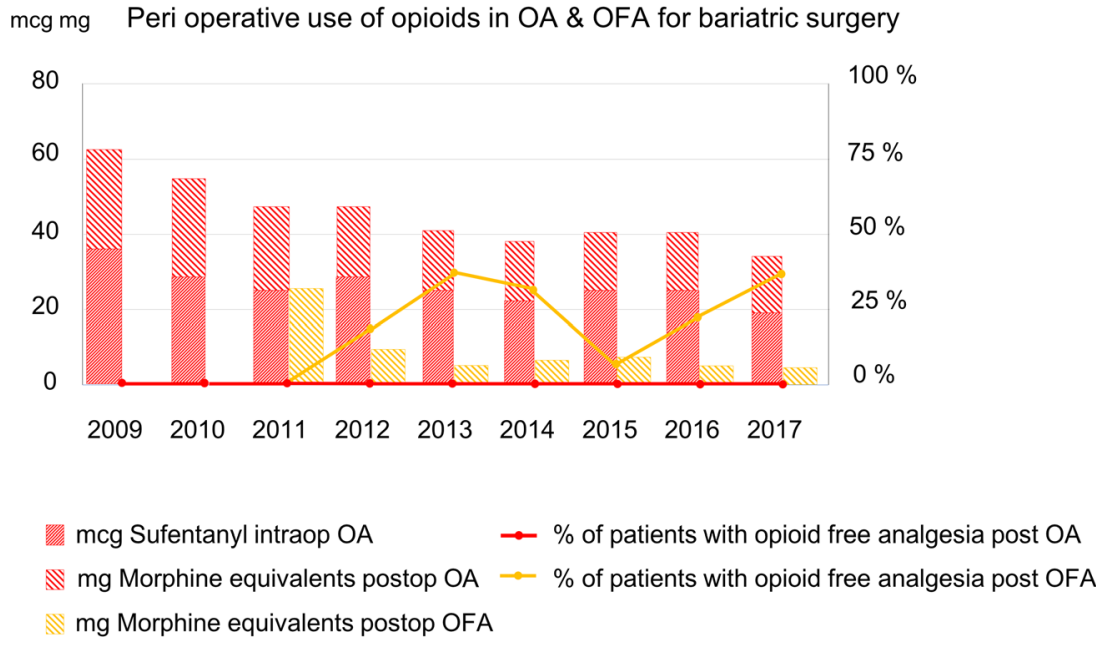

mcg mg Peri operative use of opioids in OA \& OFA for bariatric surgery 
non-continuous clinical deep NMB group $(166.53 \mathrm{~cm}$ vs. $168.78 \mathrm{~cm} p<0.001$ ).

Surgical team experience was linked to the year of surgery and represented the time-dependent effects that might impact surgical outcomes. Anaesthesiologists and residents-intraining change over time; some have worked on many cases, whereas others have only experienced a small number of cases. Not every anaesthesiologist has used the same number of continuous clinical deep NMB and OFA. Therefore, we accounted for the experience of anaesthesiologists as the number of bariatric procedures performed in the last 12 months by each anaesthesiologist or resident (under supervision).

Our levelled logistic regression analysis used a very large database, allowing us to correct for the effects of many factors that co-contributed to an outcome. We separated the impact of bariatric anaesthesia experience by first levelling by the number of bariatric cases each anaesthesiologist had done that year. Levelling first (instead of adding experience as a covariant) eliminates the potential effects of experienced anaesthesiologists performing more deep NMB or OFA. This was needed as bariatric anaesthesia experience improved outcomes $(p<0.001)$ as seen in Table 6.

OFA, continuous clinical deep NMB, younger patient age, greater surgical team experience, and surgery type (primary case) were all independently associated with fewer perioperative complications. Only one death occurred within a month of surgery out of more than 9000 patients, indicating the high quality of care received by all patients. However, this very low number prevents the use of mortality as an outcome for further analysis.

Levelled logistic regression analysis showed that continuous clinical deep NMB was not associated with any of the outcomes related to the utilization of healthcare resources. It is likely that the numbers of reoperations and readmissions were too low to detect significant differences. Before 2009, admissions to a high-dependency care unit were affected by the possibility of insufficient NMB reversal due to the unavailability of sugammadex and limited NMB monitoring. All patients after this time (i.e., included in the current study) underwent neuromuscular transmission monitoring at the end of surgery and were reversed with $2 \mathrm{mg} / \mathrm{kg}$ or more sugammadex, if necessary. This practice may have also contributed to the low number of complications since 2009. We found that deep NMB facilitated intra-operative ventilation with permissive hypercapnia when no opioid-induced respiratory depression was available.

A combination of less pain, less PONV, faster recovery, and improved mobilization after OFA might partially explain the improved outcomes after OFA.

The dose of post-operative opioids dropped from 30 to $15 \mathrm{mg}$ morphine equivalents in the OA group. This could be due to a reduction in the total dose of sufentanil used intraoperatively and the more frequent intra-operative use of an additive. The total amount of post-operative opioids dropped in the OFA groups to approximately $5 \mathrm{mg}$ morphine equivalent.

When use of a high intra-operative dose of dexmedetomidine was reduced in 2015, fewer patients received OFA post-operatively. Only in the last few years, the number of OFA patients has increased again due to the continuing use of a post-operative infusion of dexmedetomidine, lidocaine, and ketamine at very low doses.

There was a strong relationship between perioperative opioid use and the number of complications, suggesting the importance of OFA.

\section{Conclusion}

Continuous clinical deep NMB and OFA were independently associated with fewer complications after bariatric surgery. Other contributing factors included patient age, surgical and anaesthesia team experience, and surgery type. OFA was associated with less healthcare resource utilization. Reduced LOS was also associated with a younger patient age, surgical team experience, and surgery type.

\section{Compliance with Ethical Standards}

Conflict of Interest Dr. Mulier reports grants from Merck, during the conduct of the study to allow independent data nurses gather retrospective clinical data and add anaesthetic data to the clinical database; he reports also personal fees and non-financial support from Merck and Johnson \& Johnson outside the submitted work. Dr. Dillemans reports personal fees and non-financial support from Johnson \& Johnson, personal fees and non-financial support from Medtronic, outside the submitted work.

Ethical Statement All procedures performed in studies involving human participants were in accordance with the ethical standards of the institutional and/or national research committee and with the 1964 Helsinki declaration and its later amendments or comparable ethical standards. The study was approved by the ethical committee of the AZ Sint Jan Brugge-Oostende hospital.

Consent Statement Since this is a retrospective study, formal consent is not required for this type of study.

Open Access This article is distributed under the terms of the Creative Commons Attribution 4.0 International License (http:// creativecommons.org/licenses/by/4.0/), which permits unrestricted use, distribution, and reproduction in any medium, provided you give appropriate credit to the original author(s) and the source, provide a link to the Creative Commons license, and indicate if changes were made.

\section{References}

1. Dillemans B, Sakran N, Van Cauwenberge S, et al. Standardization of the fully stapled laparoscopic Roux-en-Y gastric bypass for obesity reduces early immediate postoperative morbidity and mortality: a single center study on 2606 patients. Obes Surg. 2009;19:1355-64. 
2. Aceto P, Modesti C, Sacco T, et al. Patient-related factors predicting workspace conditions during laparoscopic bariatric surgery. Obes Surg. 2018;28:3172-6.

3. Madsen MV, Staehr-Rye AK, Gätke MR, et al. Neuromuscular blockade for optimizing surgical conditions during abdominal and gynaecological surgery: a systematic review. Acta Anaesthesiol Scand. 2015;59:1-16.

4. Mulier J, Dillemans B. Deep neuromuscular blockade versus remifentanil or sevoflurane to augment measurable laparoscopic workspace during bariatric surgery analysed by a randomized controlled. Trial J Clin Anesth Pain Med. 2018;2:014.

5. Bruintjes MH, van Helden EV, Braat AE, et al. Deep neuromuscular block to optimize surgical space conditions during laparoscopic surgery: a systematic review and meta-analysis. Br J Anaesth. 2017;118:834-42.

6. Blobner M, Frick C, Stauble R, et al. Neuromuscular blockade improves surgical conditions (NISCO). Surg Endosc. 2015;29: 627-36.

7. Koninckx PR, Gomel V, Ussia A, et al. Role of the peritoneal cavity in the prevention of postoperative adhesions, pain, and fatigue. Fertil Steril. 2016;106:998-1010.

8. Thorell A, MacCormick A, Awad S, et al. Guidelines for perioperative care in bariatric surgery: Enhanced Recovery After Surgery (ERAS) society recommendations. World J Surg. 2016;40:206583.

9. Lavand'homme P, Steyaert A. Opioid-free anesthesia opioid side effects: tolerance and hyperalgesia. Best Pract Res Clin Anaesthesiol. 2017;31:487-98.

10. Hayhurst CJ, Durieuw ME. Differential opioid tolerance and opioid-induced hyperalgesia. Anesthesiology. 2016;124:483-8.

11. Roberts GW, Bekker TB, Carlsen HH, et al. Postoperative nausea and vomiting are strongly influenced by postoperative opioid use in a dose-related manner. Anesth Analg. 2005;101:1343-8.

12. Özdemir-van Brunschot DMD, Scheffer GJ, van der Jagt M, et al. Quality of recovery after low-pressure laparoscopic donor nephrectomy facilitated by deep neuromuscular blockade: a randomized controlled study. World J Surg. 2017;41:2950-8.

13. Chung F, Yang Y, Liao P. Predictive performance of the STOPBang score for identifying obstructive sleep apnea in obese patients. Obes Surg. 2013;23:2050-7.

14. Mary Lynn McPherson Demystifying opioid conversion calculations: a guide for effective dosing. ISBN 978-1-58528-198-5 2009 American Society of Health-System Pharmacists, Inc.
15. Mulier J, Wouters R, Dillemans B, et al. A randomized controlled, double-blind trial evaluating the effect of opioid-free versus opioid general anaesthesia on post-operative pain and discomfort measured by the QoR-40. J Clin Anesth Pain Med. 2018;2:015.

16. Gallart L, Canet J. Post-operative pulmonary complications: understanding definitions and risk assessment. Best Pract Res Clin Anaesthesiol. 2015;29:315e330.

17. Mulier J, Dubois P. A review of the interest of sugammadex for deep neuromuscular blockade management in Belgium. Acta Anaesthsiol Belg. 2013;64:49-60.

18. Moerer O, Bittner J, Hinz J, et al. Effect of rocuronium on the diaphragm, musculus adductor pollicis and orbicularis oculi in two groups of different age. Anasthesiol Intensivmed Notfallmed Schmerzther. 2005;40:217-24.

19. Rosenberg J, Herring WJ, Blobner M, et al. Deep neuromuscular blockade improves laparoscopic surgical conditions: a randomized, controlled study. Adv Ther. 2017;34:925-36.

20. Baykara N, Sahin T, Alpar R, et al. Evaluation of intense neuromuscular blockade caused by rocuronium using posttetanic count in male and female patients. J Clin Anesth. 2003;15:446-50.

21. Rotava P, Cavalcanti IL, Barrucand L, et al. Effects of magnesium sulphate on the pharmacodynamics of rocuronium in patients aged 60 years and older: a randomised trial. Eur J Anaesthesiol. 2013;30: 599-604.

22. Moerer O, Bittner J, Hinz J, et al. Neuromusculare Wirkzeiten von Rocuronium am diaphragma, Musculus adductor pollicis und orbicularis oculi in zwei altersgruppen. Anaesthesiol Intensivmed Notfallmed Schmerzther. 2005;40:217-24.

23. Stourac P, Adamus M, Seidlova D, et al. Low-dose or high-dose rocuronium reversed with neostigmine or sugammadex for cesarean delivery anesthesia: a randomized controlled noninferiority trial of time to tracheal intubation and extubation. Anesth Analg. 2016;122:1536-45.

24. Gurusamy K, Vaughan J, Davidson BR. Low pressure versus standard pressure pneumoperitoneum in laparoscopic cholecystectomy. Cochrane Database of Systematic Reviews 2014, Issue 3. Art. No.: CD006930.

Publisher's Note Springer Nature remains neutral with regard to jurisdictional claims in published maps and institutional affiliations. 